\title{
Evaluation of Wing Load Calibration and Sensing Methods Using Conventional Strain Gages and a Fiber Optic Sensing System Installed on a Straight Tapered Wing
}

\author{
Eric J. Miller, Frank Pena, Ashante Jordan, Larry Hudson
}

NASA Armstrong Flight Research Center, Edwards, California, 93523

William Lokos

Jacobs Engineering, NASA Armstrong Flight Research Center, Edwards Air Force Base, California 93523 


\section{Outline}

- Introduction

- Historical Examples

- Structural Sensing Methods

- Linear Regression Methods

- Operational Loads Estimation Algorithm

- Instrumentation

- Conventional Strain Gages

- Fiber Optic Strain Sensors

- Load Cases

- Test Results

- Conclusions

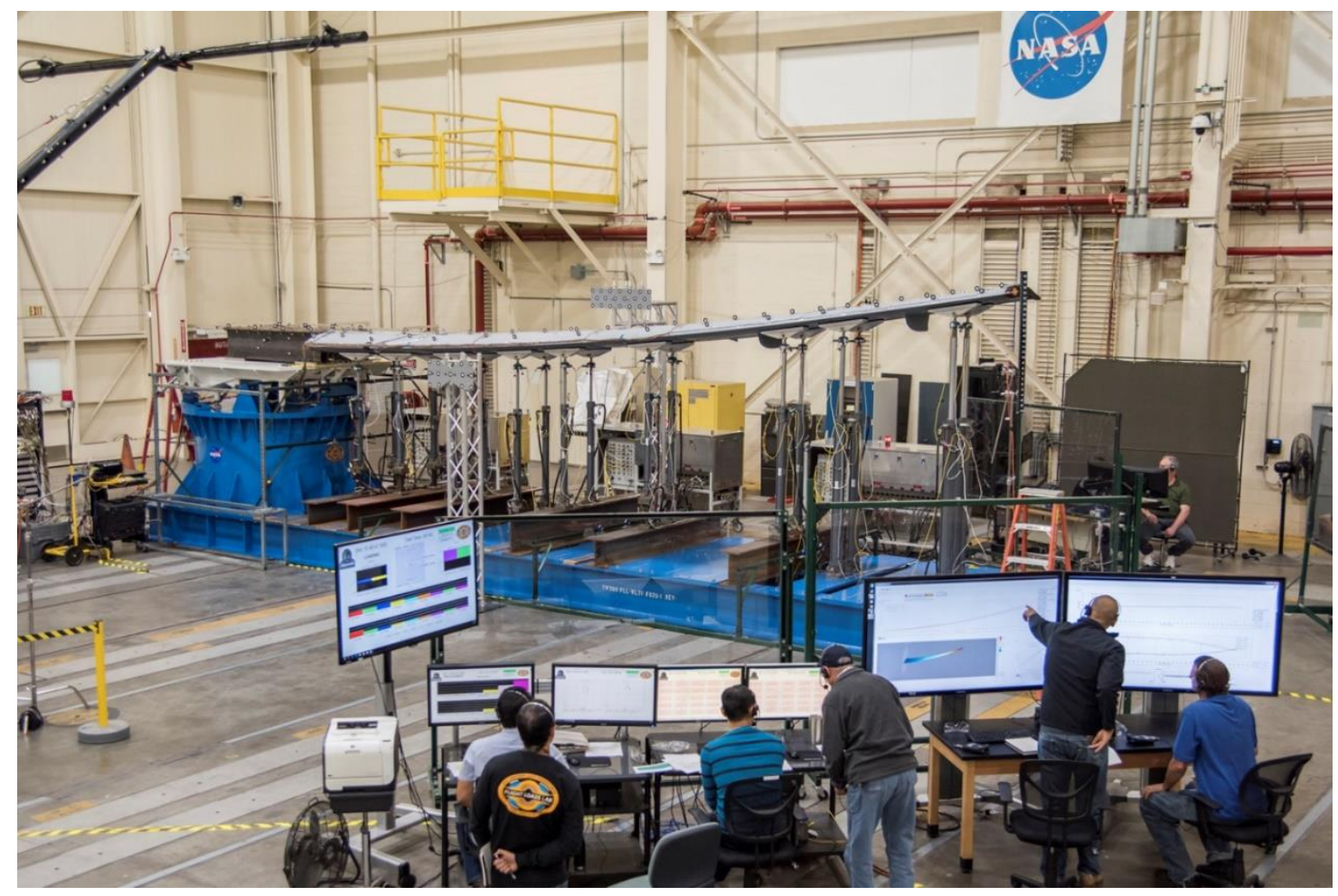




\section{Introduction}

- Reasons for monitoring structural loads

1. Airworthiness Clearance - Confidence in the loads for envelope clearance, do the loads match predictions?

2. Health Monitoring - Insight into the operational loads environment allows for more informed inspections and maintenance decisions

3. Structural Load Alleviation - Reducing the internal loading during maneuvers or gust encounters provide protection against structural overload

4. Structural Optimization / Model Validation - New structures are being developed and the models need to be validated in relevant environments

5. Aerodynamic Model Validation - The measured structural loads with inertial correction can be used to validate computational fluid dynamic (CFD) models

- It is important to understand what information is required before selecting the sensors and load monitoring methodology 


\section{Introduction}

- New instrumentation techniques have been developed that allow a much greater number of sensors to be monitored thus allowing greater insight into the structural response of structures in-flight

- A straight tapered wing with 30ft semi span was instrumented with both conventional strain gages and fiber optic strain sensors

- A conventional loads calibration was conducted on the wing, known loads were applied to the wing and strain gage and fiber optic strain sensor data was recorded

- The loads test program was named the Calibration Research Wing or CREW Loads Test, it also served as a pathfinder for the Passive Aeroelastic Tailored Wing Testing

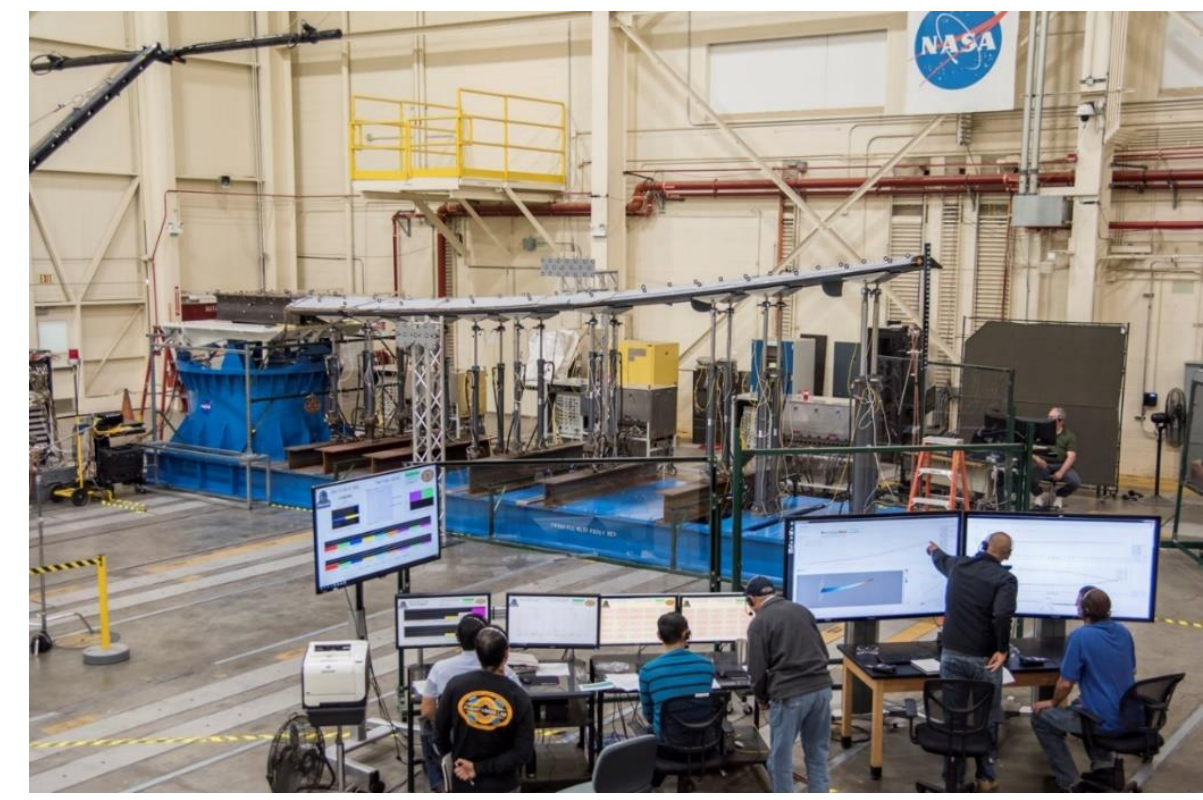




\section{Historical Examples}

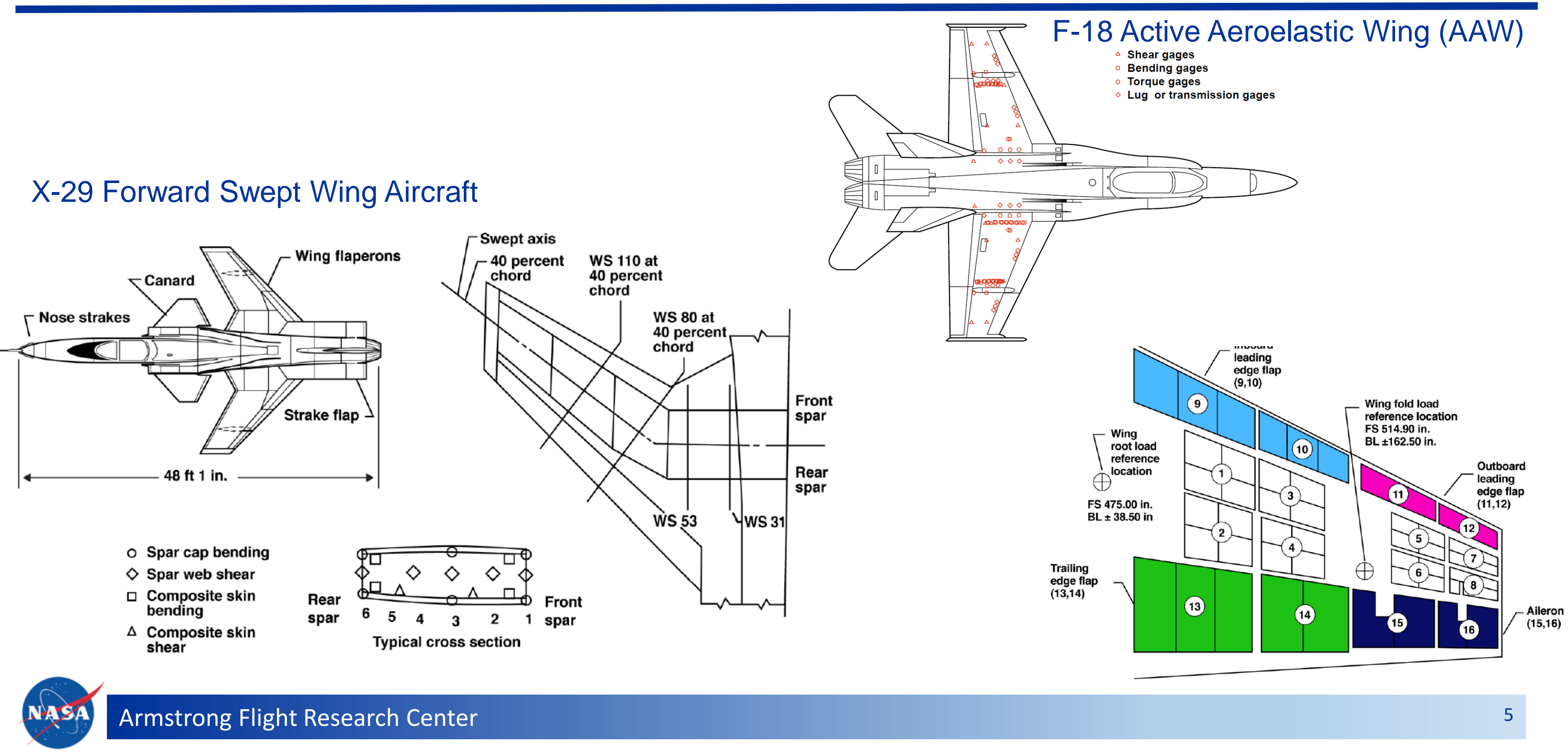




\section{Structural Sensing Methods}

- Methods discussed in this presentation:

- Linear regression methods

- Operational Loads Estimation Algorithm

- Other Methods:

- Photogrammetry

- Flight calibration methods

- Finite element methods 


\section{Structural Load Sensing Scheme}

Conventional scheme (strain gages located at a single spanwise station) provides ability to monitor loads at single spanwise station.

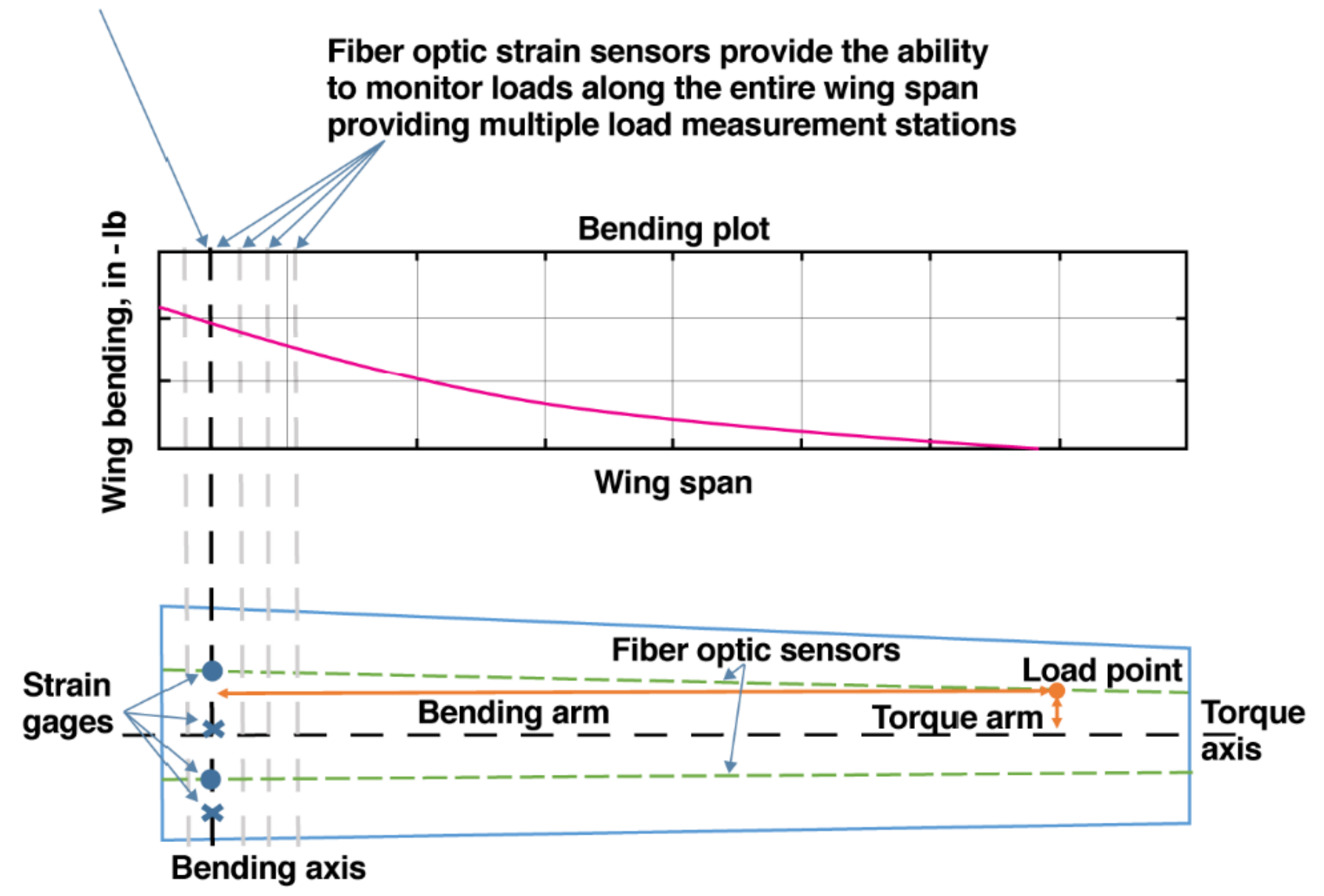

Spar cap axial strain gage

Spar web rosette strain gage

Wing skin rosette strain gage

Spar cap axial fiber optic strain sensor

Wing skin rosette fiber optic strain sensor

Typical wing cross section

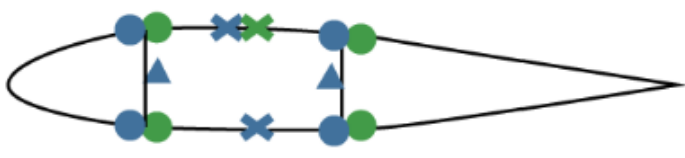




\section{Methods - Linear Regression}

- Strain gage, fiber optic strain sensing, and load data is collected during three load cycles

- The linear portion of cycle 2 or 3 (green arrow) is taken from the dataset for calibration

\begin{tabular}{|c|c|c|c|c|c|c|c|}
\hline \hline $\begin{array}{c}\text { Load } \\
\text { measurement }\end{array}$ & $\begin{array}{c}\text { Applied } \\
\text { load }\end{array}$ & $\begin{array}{c}\text { Gage A } \\
\text { output }\end{array}$ & $\begin{array}{c}\text { Gage B } \\
\text { output }\end{array}$ & $\begin{array}{c}\text { Gage C } \\
\text { output }\end{array}$ & $\begin{array}{c}\text { Gage D } \\
\text { output }\end{array}$ & $\cdots$ & $\begin{array}{c}\text { Gage n } \\
\text { output }\end{array}$ \\
\hline 1 & $\mathrm{~V}_{1}$ & $\mu_{1, \mathrm{~A}}$ & $\mu_{1, \mathrm{~B}}$ & $\mu_{1, \mathrm{C}}$ & $\mu_{1, \mathrm{D}}$ & $\ldots$ & $\mu_{1, \mathrm{n}}$ \\
\hline 2 & $\mathrm{~V}_{2}$ & $\mu_{2, \mathrm{~A}}$ & $\mu_{2, \mathrm{~B}}$ & $\mu_{2, \mathrm{C}}$ & $\mu_{2, \mathrm{D}}$ & $\ldots$ & $\mu_{2, \mathrm{n}}$ \\
\hline 3 & $\mathrm{~V}_{3}$ & $\mu_{3, \mathrm{~A}}$ & $\mu_{3, \mathrm{~B}}$ & $\mu_{3, \mathrm{C}}$ & $\mu_{3, \mathrm{D}}$ & $\ldots$ & $\mu_{3, \mathrm{n}}$ \\
\hline$\ldots$ & $\ldots$ & $\ldots$ & $\ldots$ & $\ldots$ & $\ldots$ & $\ldots$ & $\ldots$ \\
\hline $\mathrm{m}$ & $\mathrm{V}_{\mathrm{m}}$ & $\mu_{\mathrm{m}, \mathrm{A}}$ & $\mu_{\mathrm{m}, \mathrm{B}}$ & $\mu_{\mathrm{m}, \mathrm{C}}$ & $\mu_{\mathrm{m}, \mathrm{D}}$ & $\ldots$ & $\mu_{\mathrm{m}, \mathrm{n}}$ \\
\hline \hline
\end{tabular}

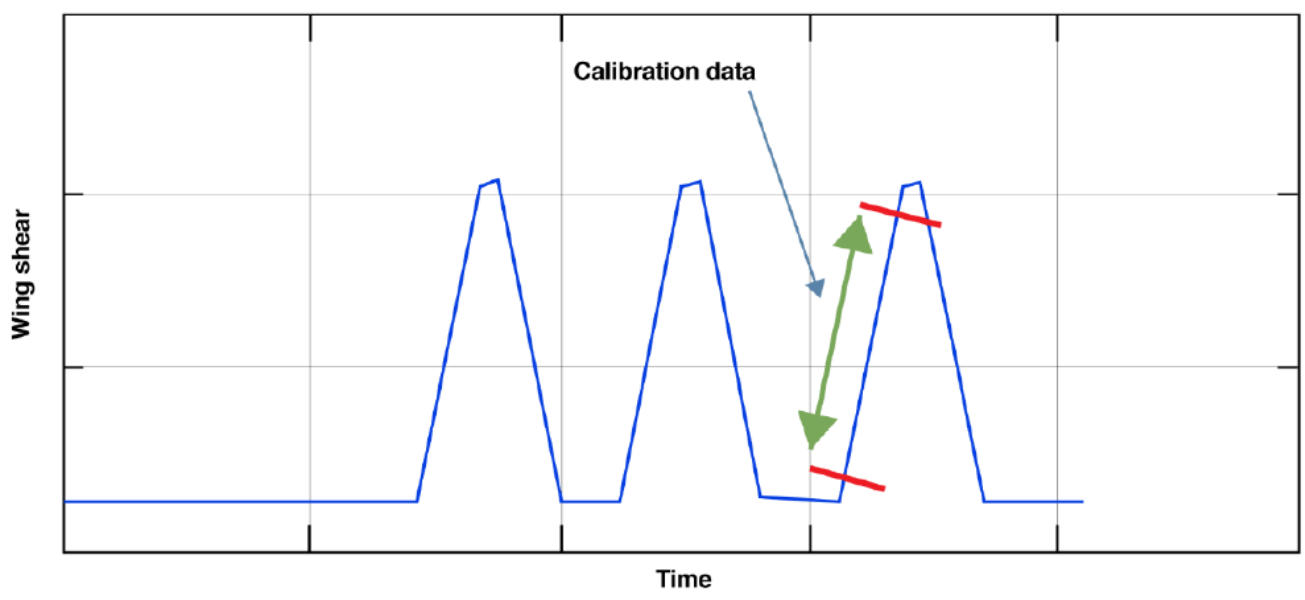

- Example: 3-gage equation that uses gages $A, C$, and $D$ and shear load V

$$
\left[\begin{array}{cccc}
1 & \mu_{1, \mathrm{~A}} & \mu_{1, \mathrm{C}} & \mu_{1, \mathrm{D}} \\
1 & \mu_{2, \mathrm{~A}} & \mu_{2, \mathrm{C}} & \mu_{2, \mathrm{D}}^{2} \\
1 & \mu_{3, \mathrm{~A}} & \mu_{3, \mathrm{C}} & \mu_{3, \mathrm{D}} \\
\cdots & \cdots & \cdots & \cdots \\
1 & \mu_{\mathrm{m}, \mathrm{A}} & \mu_{\mathrm{m}, \mathrm{C}} & \mu_{\mathrm{m}, \mathrm{D}}
\end{array}\right]\left[\begin{array}{c}
\beta_{0} \\
\beta_{1} \\
\beta_{2} \\
\beta_{3}
\end{array}\right]=\left[\begin{array}{c}
V_{0} \\
V_{1} \\
V_{2} \\
\cdots \\
V_{m}
\end{array}\right]
$$

- The least squares solution for the coefficients $\beta$ can then be found

$$
\left[\mu_{j, k}\right]\left[\beta_{k}\right]=[V]
$$




\section{Methods - Operational Loads Estimation Algorithm}

- Moment-strain relationship of the classical beam $\frac{d^{2} z(y)}{d y^{2}}=\frac{M(y)}{E(y) I(y)}$

- Bending stress and normal stress strain relationship

$$
\sigma_{\text {upper }}(y)=\frac{M(y) \cdot c_{\text {upper }}(y)}{I(y)}=E(y) \cdot \varepsilon_{\text {upper }}(y) \Rightarrow \frac{M(y)}{E(y) I(y)}=\frac{\varepsilon_{\text {upper }}(y)}{c_{\text {upper }}(y)}
$$

- Distance to the neutral axis

$$
c_{\text {upper }}(y)=\frac{h(y) \cdot \varepsilon_{\text {upper }}(y)}{\varepsilon_{\text {lower }}(y)-\varepsilon_{\text {upper }}(y)}
$$

- Section properties of the beam structure

$$
E(y) I(y)=M(y) \cdot \frac{h(y)}{\varepsilon_{\text {lower }}(y)-\varepsilon_{\text {upper }}(y)}
$$

- A single load case is applied to the wing to calculate the section El properties

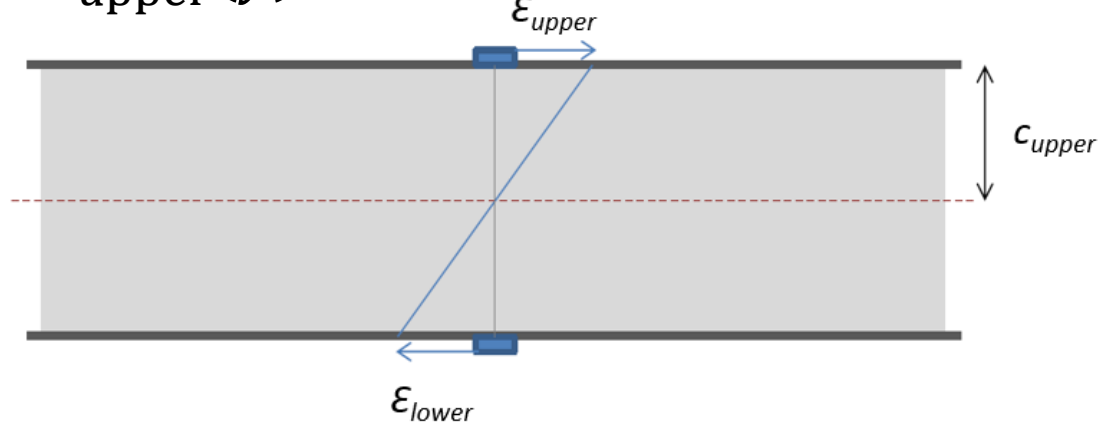




\section{Methods - Operational Loads Estimation Algorithm}

- The bending moment can be calculated by knowing the strains and section properties

- Integrating moment strain relationship to calculate slope

- The displacement can be determined by integrating the slope

- Using small angle approximation $\sin \phi \approx \phi$, the calculation for vertical displacement becomes

$$
M(y)=E(y) I(y) \cdot \frac{\varepsilon_{\text {lower }}(y)-\varepsilon_{\text {upper }}(y)}{h(y)}
$$$$
\frac{\partial z}{\partial y}=\tan (\phi) \approx \phi_{n}=\phi_{0}+\sum_{i=1}^{n-1}\left(\left(\frac{M}{E I}\right)_{i}+\left(\frac{M}{E I}\right)_{i+1}\right) \cdot \frac{\Delta L}{2}
$$$$
Z_{n}=Z_{0}+\sum_{i=1}^{n} \sin \left(\phi_{i}\right) \cdot \Delta L_{i}
$$

$$
Z_{O L E A}=Z_{0}+\sum_{i=1}^{n} \phi_{i} \cdot \Delta L_{i}
$$




\section{Methods - Operational Loads Estimation Algorithm}

- Initial Values:

- The wing displacement ZOLEA is calculated based on section properties and strains

- Initial guess for shear load $\left(\mathrm{V}_{\text {Model }}\right)$ is provided

- The shear load $\left(\mathrm{V}_{\text {Model }}\right)$ is integrated 3 times to get a wing displacement $\left(Z_{\text {Model }}\right)$

- The $Z_{\text {Model }}$ is compared to the $Z_{\text {OLEA }}$

- If $Z_{\text {Model }}$ is not within an error threshold, the $V_{\text {Model }}$ is multiplied by a correction factor and the model integration loop is repeated

- If $Z_{\text {Model }}$ is within the error threshold

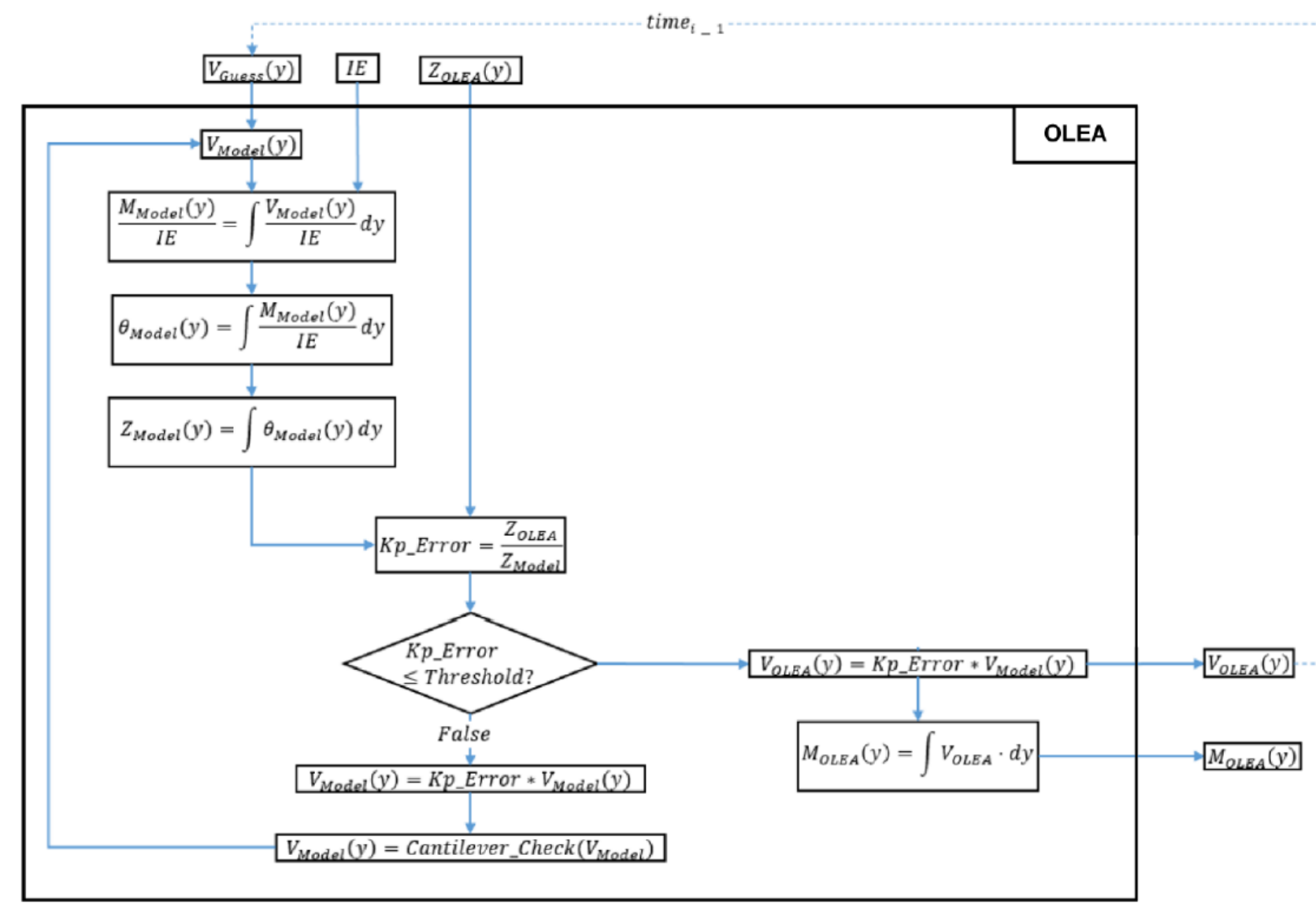
- $\mathrm{V}_{\text {OLEA }}$ and $\mathrm{M}_{\text {OLEA }}$ are then calculated 


\section{Test Article - Instrumentation}

- Foil strain gages

- 5 spanwise stations with eight full-bridges per station

- Eight internal full-bridge gages

- 14 quarter-bridge gages co-located along optical fibers
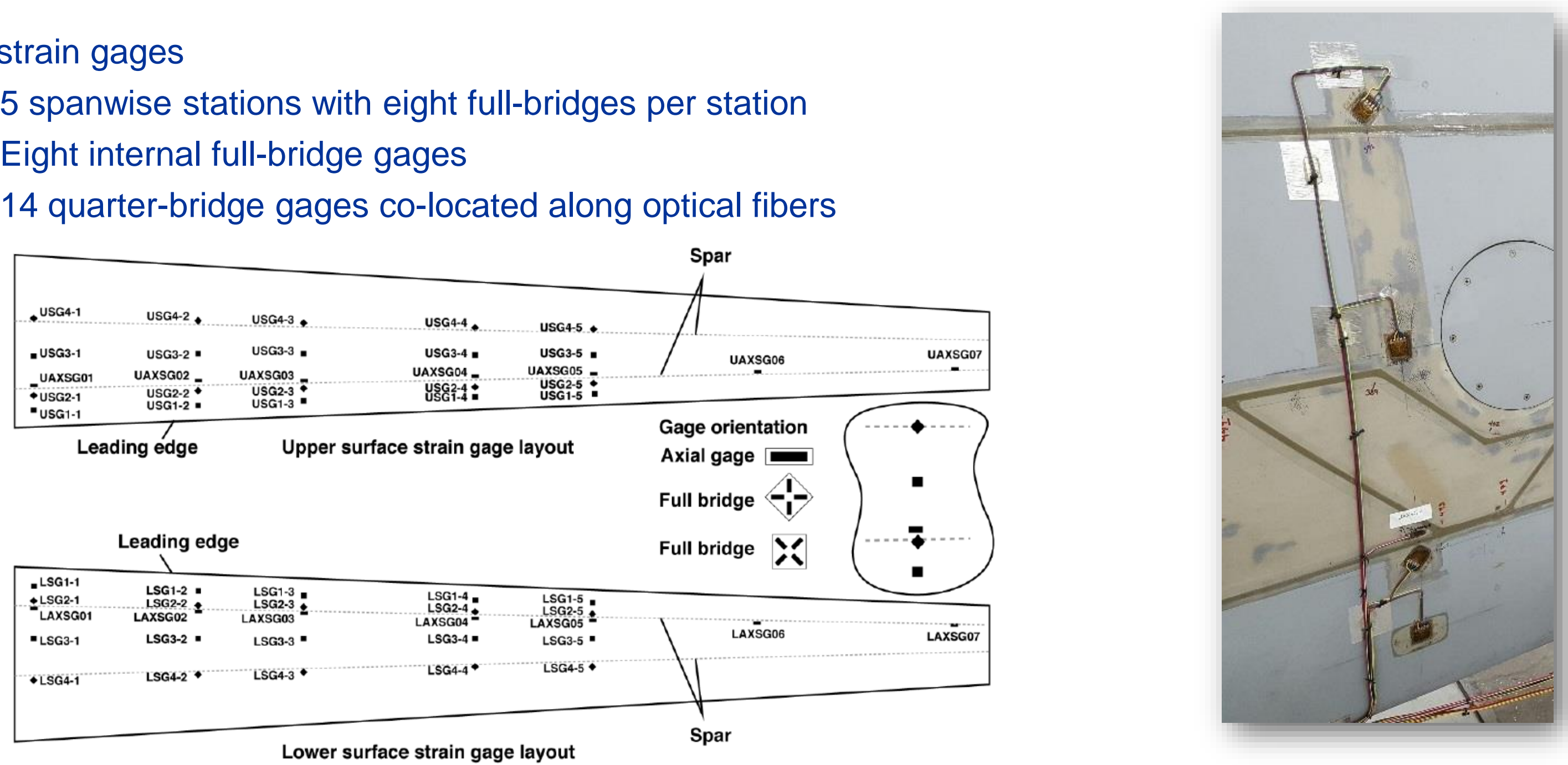


\section{Test Article - Instrumentation}

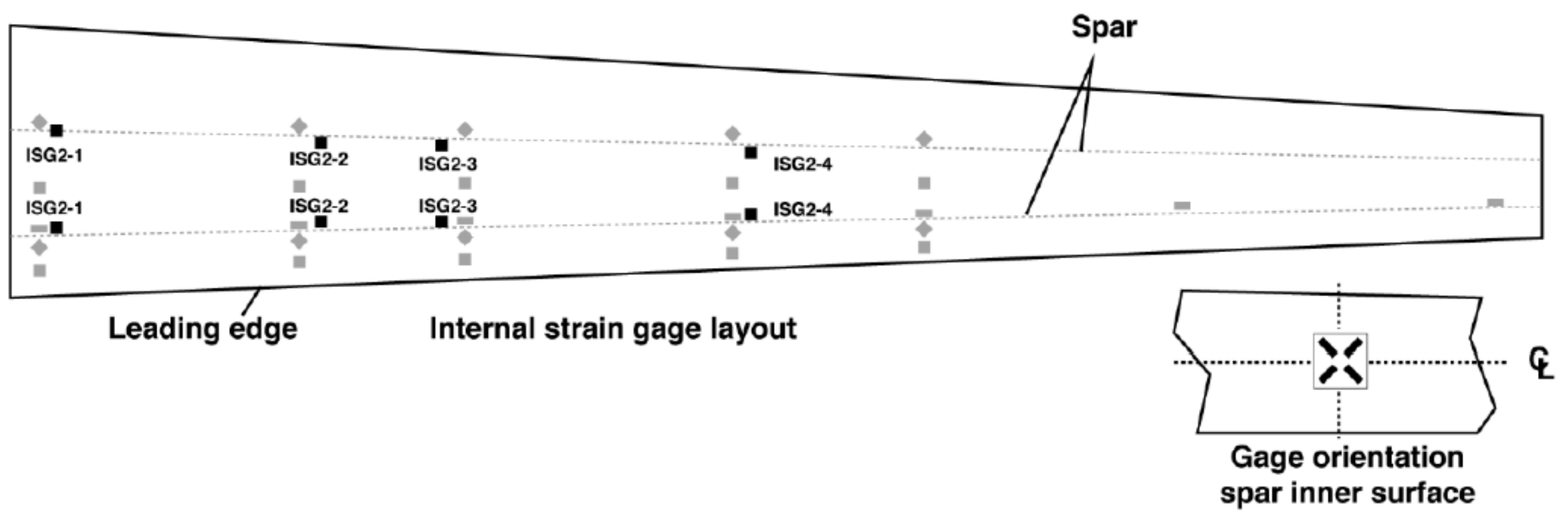




\section{Test Article - Instrumentation}

- Fiber Optic Strain Sensing

- Eight optical fibers installed on upper and lower surfaces

- Each spar has a fiber on the upper and lower surfaces

- Fiber along the $40 \%$ chord on upper and lower surfaces

- Saw-tooth pattern on the upper and lower surfaces

\section{Upper Surface}

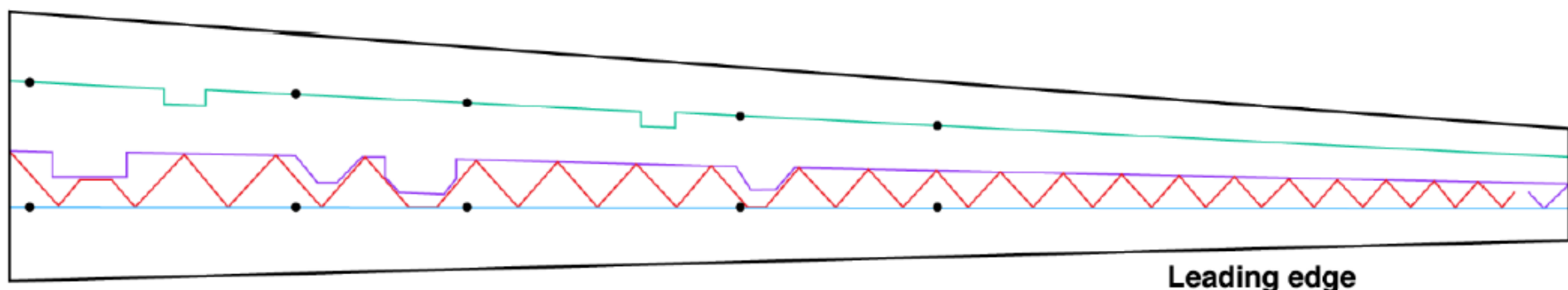

\section{Lower Surface}

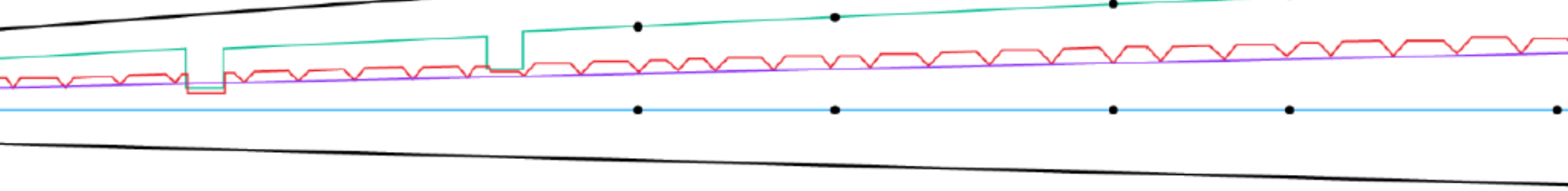

Leading edge 


\section{Test Article - Instrumentation}

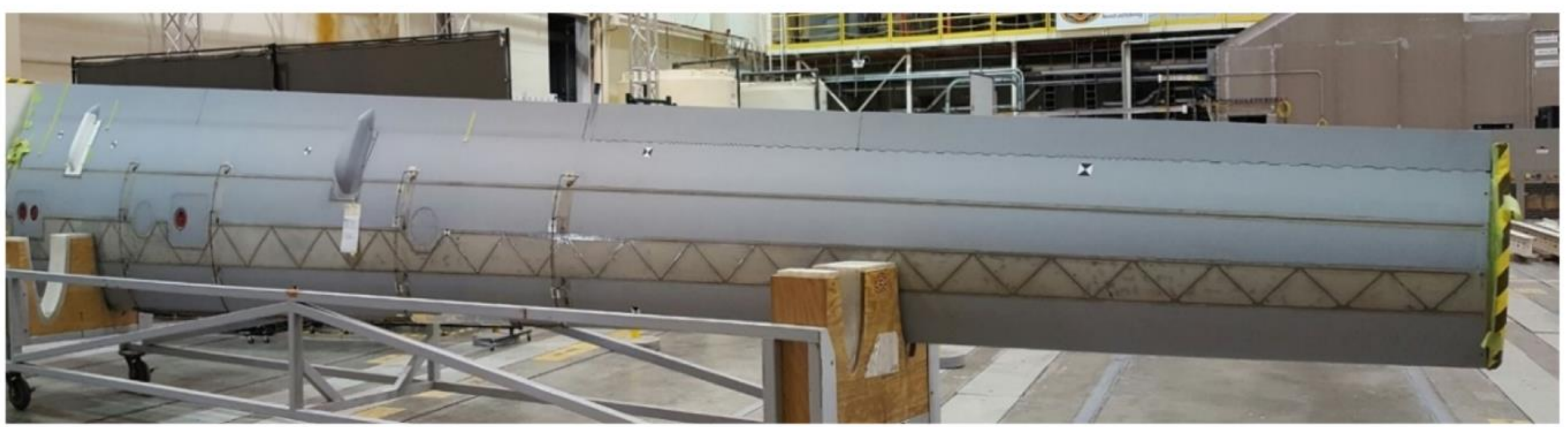




\section{Locating Instrumentation}

- Laser scan of instrumentation locations was conducted and resulting points were transferred into a FEM model

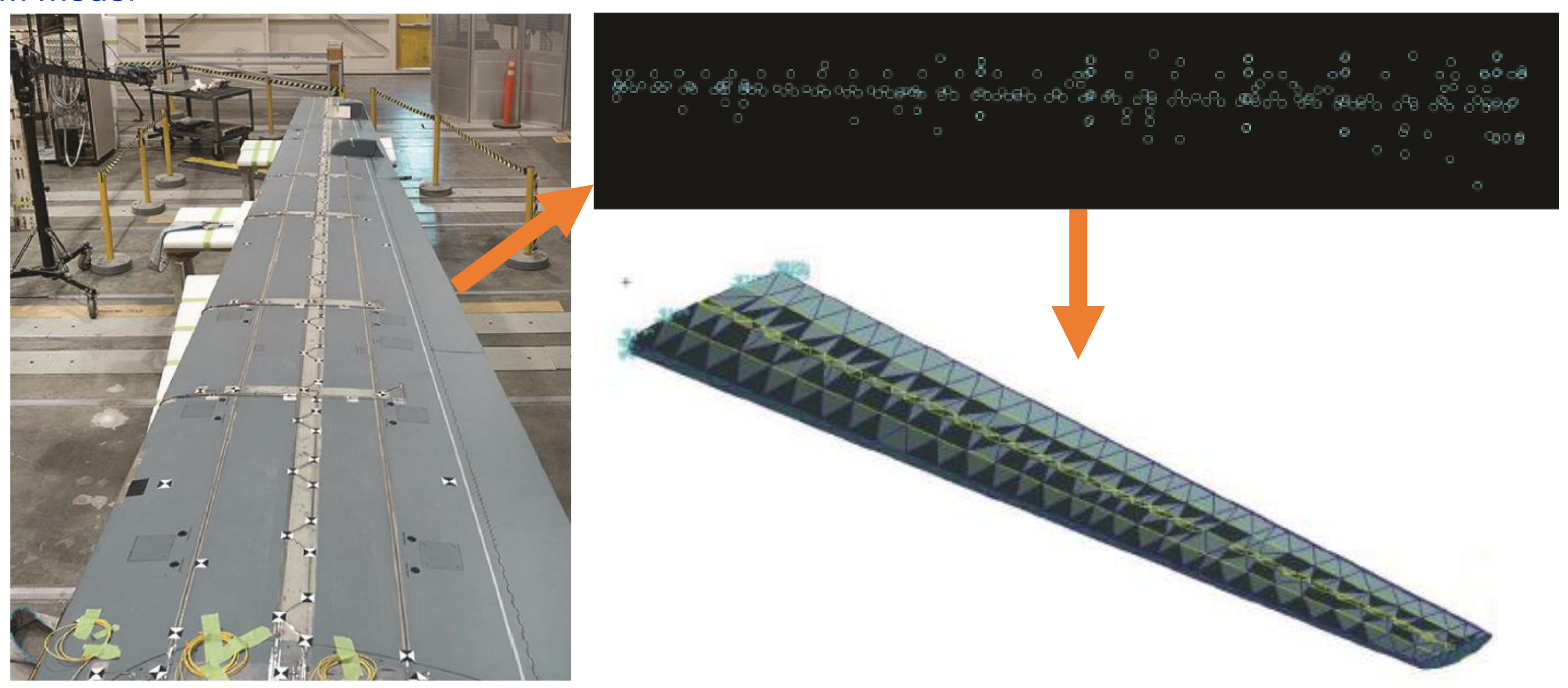




\section{Load Cases - Bending Torque Plot}

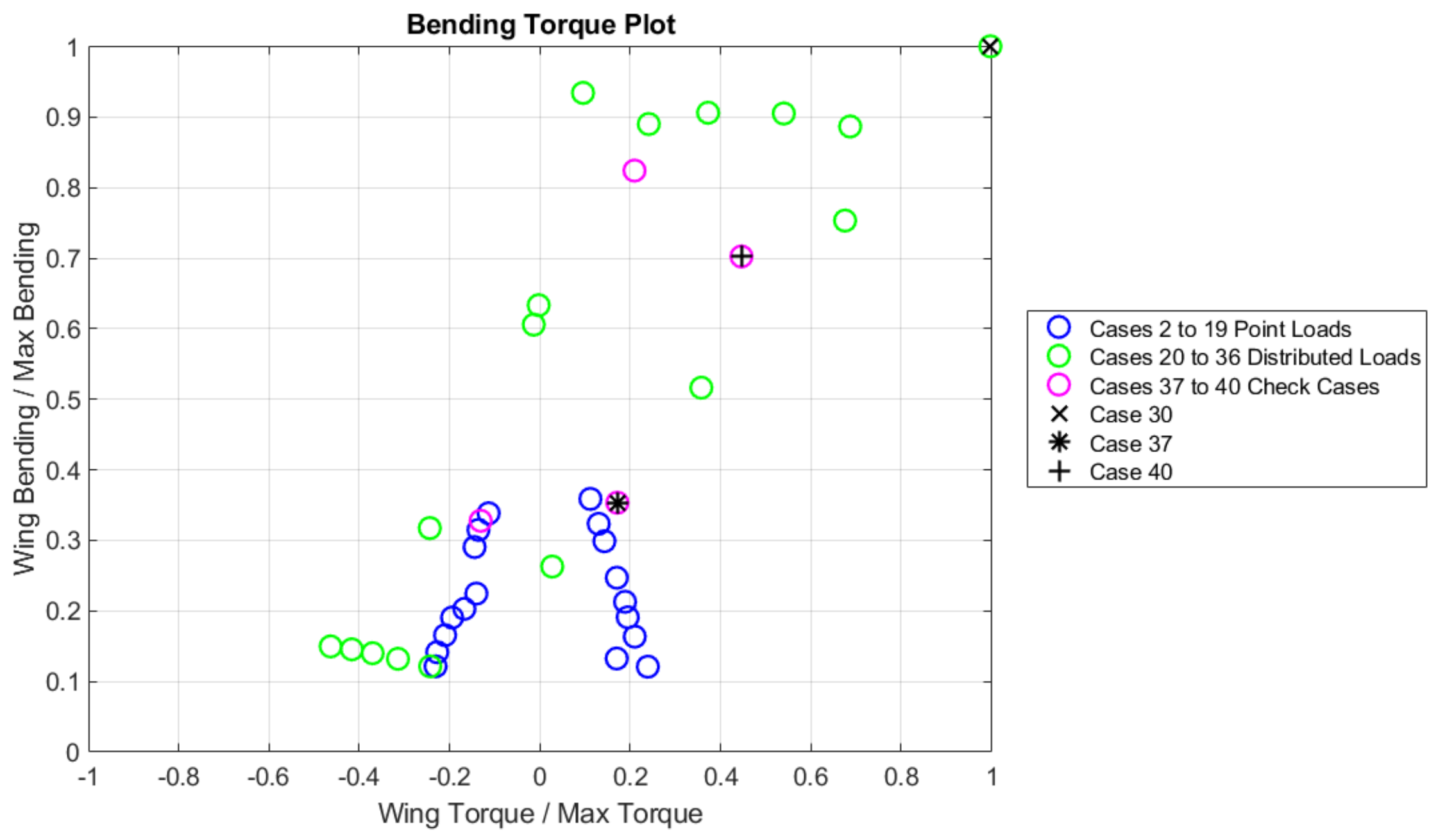




\section{Load Cases - Shear, Bending and Torque}
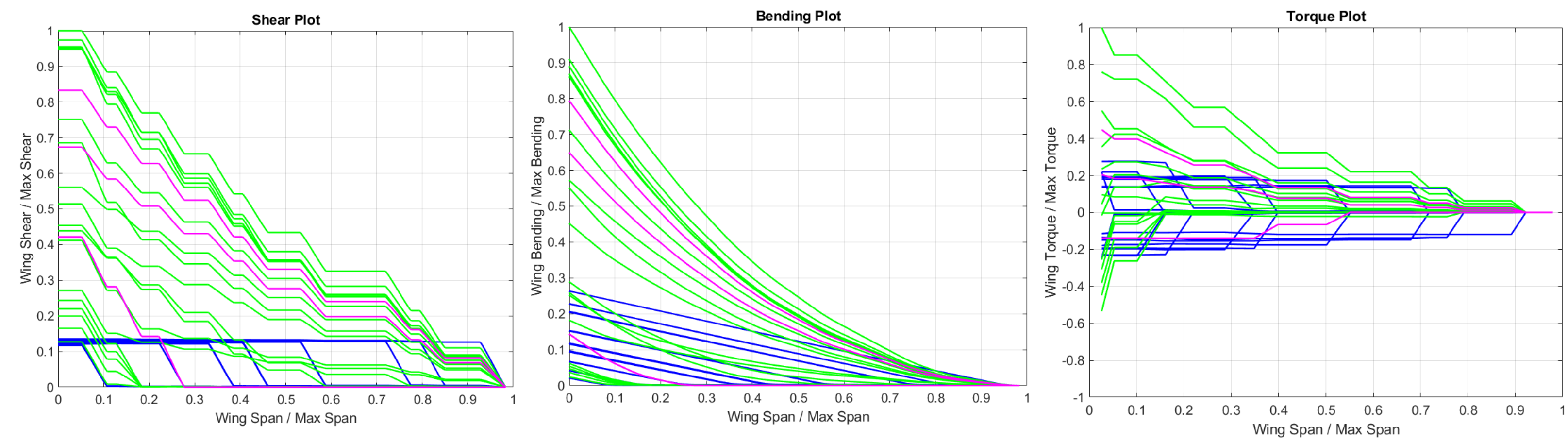


\section{Test Results}

\section{- Result Cases:}

\begin{tabular}{|l|l|l|l|l|}
\hline \hline Method & Calculated loads & Calibration cases & Check cases & Instrumentation \\
\hline Linear regression & Shear, bending, and torque & Point load cases 2-19 & Load case 37 and 40 & Strain gage \\
\hline Linear regression & Shear, bending, and torque & Distributed load cases 20-36 & Load case 37 and 40 & Strain gage \\
\hline Linear regression & Bending and torque & Point load cases 2-19 & Load case 37 and 40 & FOSS \\
\hline Linear regression & Bending and torque & Distributed load cases 20-36 & Load case 37 and 40 & FOSS \\
\hline OLEA - shape method & Shear and bending & Load case 30 & Load case 37 and 40 & FOSS \\
\hline \hline
\end{tabular}

- Error Analysis:

$$
\text { ERMS }=100 * \sqrt{\frac{\sum_{j=1}^{\mathrm{m}}\left(x^{\prime} j_{j}-x_{j}\right)^{2}}{\sum_{j=1}^{\mathrm{m}} x_{j}^{2}}} \quad \begin{aligned}
& \text { high aspect ratio straight tapered wing? } \\
& \quad \begin{array}{l}
\text { Shear: }<5 \% \\
\text { - } \text { Bending: }<5 \%
\end{array} \\
& \quad \text { Torque: }<20 \%
\end{aligned}
$$

What target errors should we expect for our 


\section{Comparison of Methods}
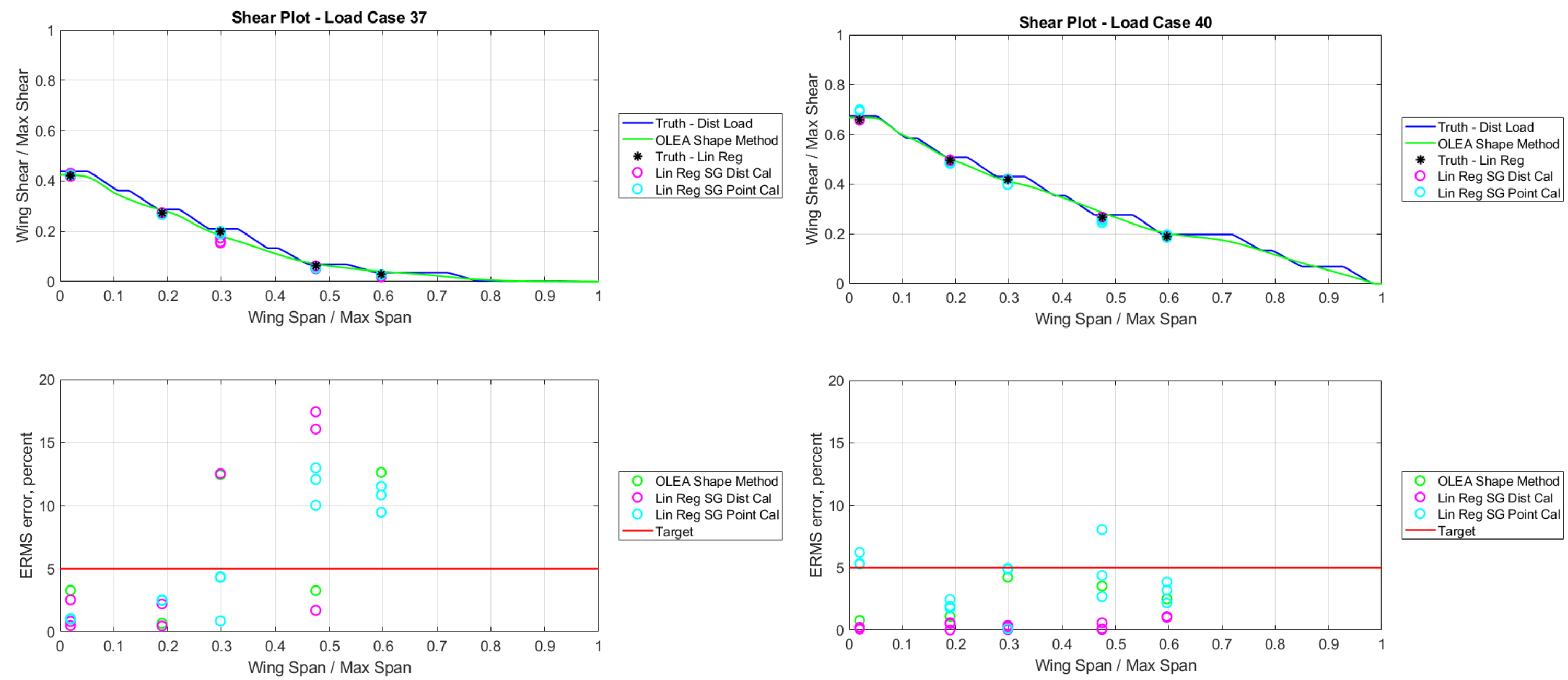

$\bigcirc$ OLEA Shape Method

Lin Reg SG Dist Cal

OLEA Shape Method

Lin Reg SG Point Ca

-Target 


\section{Comparison of Methods}
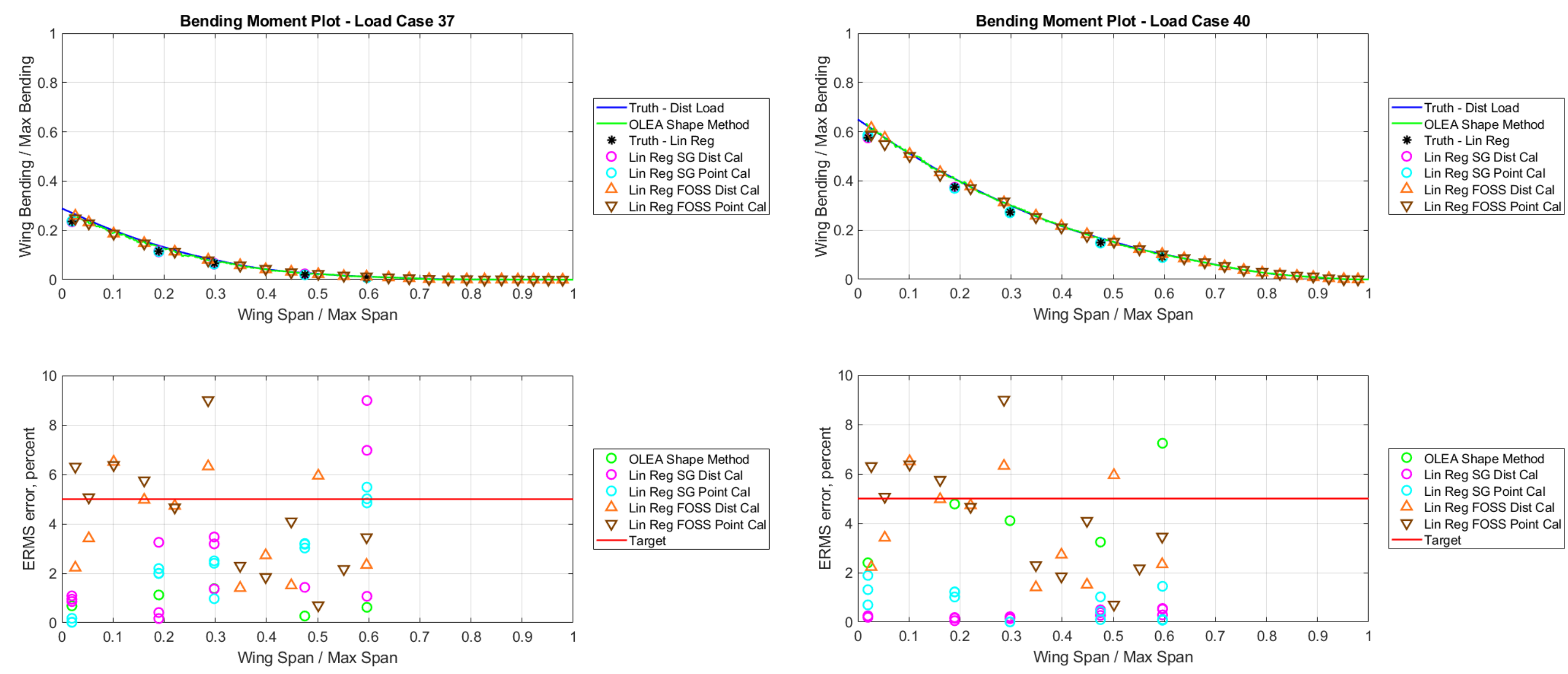


\section{Comparison of Methods}
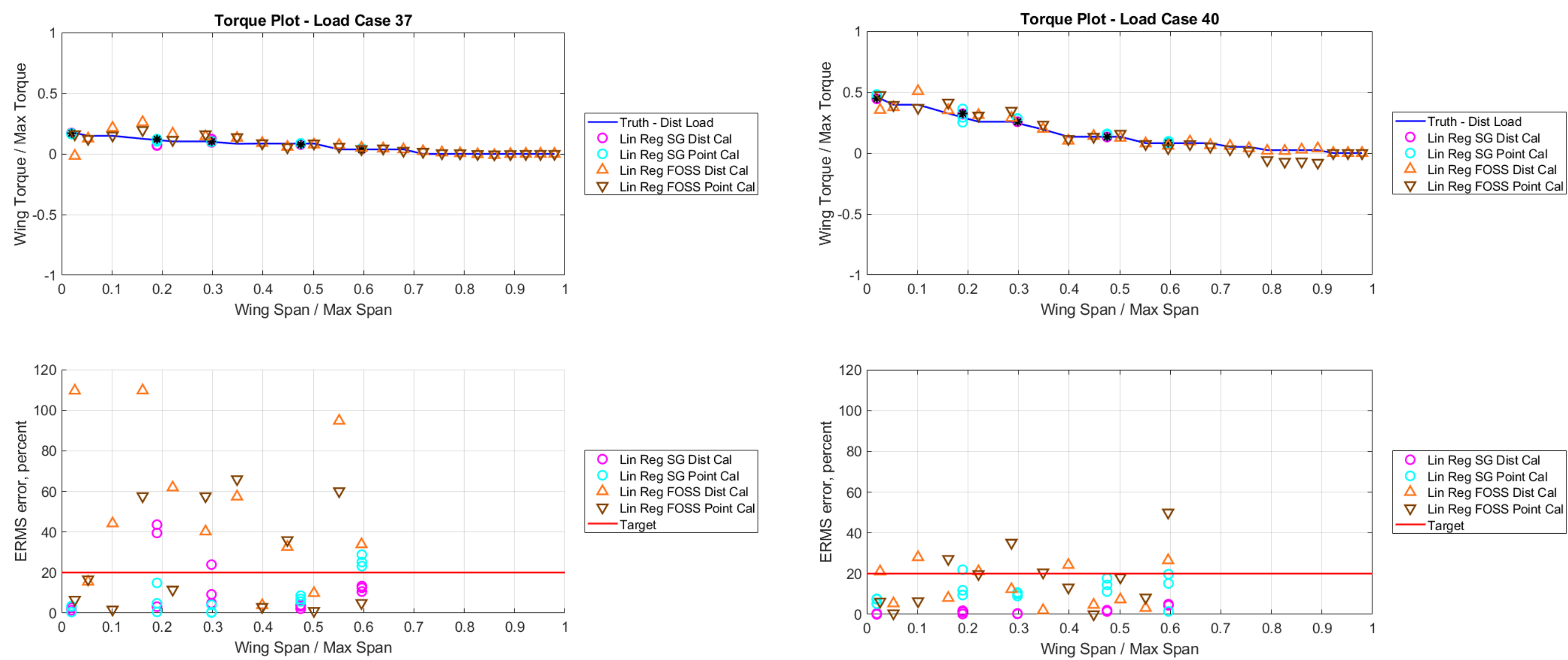

$\begin{array}{ll} & \text { Lin Reg SG Dist Cal } \\ & \text { Lin Reg SG Point Cal }\end{array}$ $\triangle$ Lin Reg FOSS Dist Cal

$\nabla$ Lin Reg FOSS Point Ca

$\nabla$ Target 


\section{Comparison of Methods}
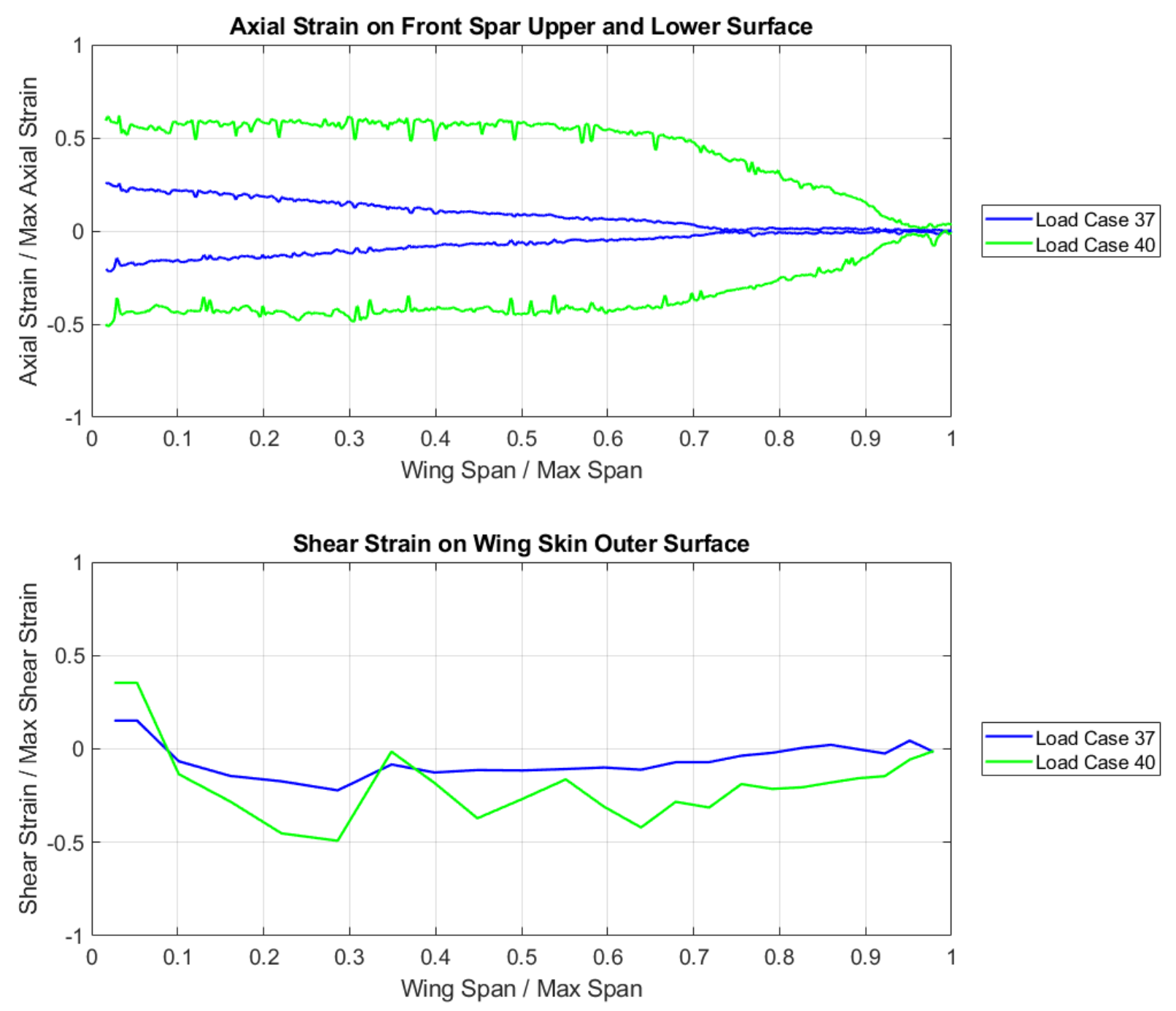


\section{Summary}

- The project requirements for load monitoring play a key role in determining which sensor and calibration method should be used

- Fiber optic measurement techniques allow for a greater number of strain sensors to be installed

- One conventional approach and two new approaches were presented for deriving sensing loads on a straight wing

- Linear regression using metallic foil strain gages

- Linear regression using FOSS

- Operational Loads Estimation Algorithm using FOSS

- Linear regression techniques can work with FOSS sensors for deriving a distributed load along the wing span resulting in greater number of load measurement stations

- New methods such as the OLEA can provide an efficient method for monitoring distributed wing shear loads using only external surface strains

- Method only requires one calibration load case, thus simplifying the load calibration test 


\section{Backup}




\section{Load Sensing Recommendations for a High Aspect Ratio Wing}

\begin{tabular}{|c|c|c|c|c|}
\hline Load type & $\begin{array}{l}\text { Recommended } \\
\text { sensor }\end{array}$ & $\begin{array}{l}\text { Strain sensor orientation and } \\
\text { location }\end{array}$ & $\begin{array}{l}\text { Recommended } \\
\text { number of load cases }\end{array}$ & $\begin{array}{l}\text { Recommended } \\
\text { calibration method }\end{array}$ \\
\hline $\begin{array}{l}\text { Shear - single wing } \\
\text { station }\end{array}$ & $\begin{array}{l}\text { Conventional } \\
\text { strain gages }\end{array}$ & $\begin{array}{l}\text { Shear rosette located on shear } \\
\text { web } \\
\text { Axial strain sensor located on } \\
\text { spar caps }\end{array}$ & $\begin{array}{l}\text { Number of load cases } \\
>\text { number of strain } \\
\text { sensors in the equation }\end{array}$ & Linear regression \\
\hline $\begin{array}{l}\text { Bending moment - } \\
\text { single wing station }\end{array}$ & $\begin{array}{l}\text { Conventional } \\
\text { strain gages }\end{array}$ & $\begin{array}{l}\text { Axial strain sensor located on } \\
\text { spar caps }\end{array}$ & 1 load case & Linear regression \\
\hline $\begin{array}{l}\text { Torque - single } \\
\text { wing station }\end{array}$ & $\begin{array}{l}\text { Conventional } \\
\text { strain gages }\end{array}$ & $\begin{array}{l}\text { Axial strain sensor located on } \\
\text { spar caps } \\
\text { Shear rosette located on shear } \\
\text { web or skins }\end{array}$ & $\begin{array}{l}\text { Number of load cases } \\
>\text { number of strain } \\
\text { sensors in the equation }\end{array}$ & Linear regression \\
\hline $\begin{array}{l}\text { Shear - distributed } \\
\text { load }\end{array}$ & FOSS sensors & $\begin{array}{l}\text { Axial strain sensor located on } \\
\text { spar caps }\end{array}$ & 1 load case & OLEA \\
\hline $\begin{array}{l}\text { Bending moment - } \\
\text { distributed load }\end{array}$ & FOSS sensors & $\begin{array}{l}\text { Axial strain sensor located on } \\
\text { spar caps }\end{array}$ & 1 load case & Linear regression \\
\hline $\begin{array}{l}\text { Torque - distributed } \\
\text { load }\end{array}$ & FOSS sensors & $\begin{array}{l}\text { Axial strain sensor located on } \\
\text { spar caps } \\
\text { Shear rosette located on shear } \\
\text { web or skins }\end{array}$ & $\begin{array}{l}\text { Number of load cases } \\
>\text { number of strain } \\
\text { sensors in the equation }\end{array}$ & Linear regression \\
\hline
\end{tabular}




\section{Discussion - Strain Gage vs FOSS Installation}

- Metallic Foil Strain Gages

- A lot of previous heritage from past projects

- Full bridge provides built-in temperature compensation

- A requirement for only a small number of strain sensors are most likely best handled by strain gages

- Fiber Optic Strain Sensors

- Capability to be multiplexed serially allowing for multiple spanwise measurements on one fiber

- Lightweight for number of strain sensors compared to weight of similar number of strain gages

- Hermetically coated glass is chemically inert, not susceptible to corrosion, and does not have potential for ground loops, electrical faults, sparking, or Joule heating. These sensors also are not negatively impacted like common aircraft avionics systems with reactions to Electro-Magnetic Interference (EMI) or ElectroMagnetic Pulses (EMP).

- Fiber can be installed much quicker than strain gages for equal number of sensors

- Fiber is much better suited to open areas and may be much better suited to observing large global effects than strain gages

- Fiber is much better suited for buckling than strain gages 